ARTICLE

\title{
Epitaxy, exfoliation, and strain-induced magnetism in rippled Heusler membranes
}

\author{
Dongxue Du', Sebastian Manzo ${ }^{1}$, Chenyu Zhang (1) ${ }^{1}$, Vivek Saraswat ${ }^{1}$, Konrad T. Genser ${ }^{2}$, Karin M. Rabe ${ }^{2}$, \\ Paul M. Voyles (1D) ${ }^{1}$, Michael S. Arnold (10 ${ }^{1} \&$ Jason K. Kawasaki (i) ${ }^{1 凶}$
}

Single-crystalline membranes of functional materials enable the tuning of properties via extreme strain states; however, conventional routes for producing membranes require the use of sacrificial layers and chemical etchants, which can both damage the membrane and limit the ability to make them ultrathin. Here we demonstrate the epitaxial growth of the cubic Heusler compound GdPtSb on graphene-terminated $\mathrm{Al}_{2} \mathrm{O}_{3}$ substrates. Despite the presence of the graphene interlayer, the Heusler films have epitaxial registry to the underlying sapphire, as revealed by x-ray diffraction, reflection high energy electron diffraction, and transmission electron microscopy. The weak Van der Waals interactions of graphene enable mechanical exfoliation to yield free-standing GdPtSb membranes, which form ripples when transferred to a flexible polymer handle. Whereas unstrained GdPtSb is antiferromagnetic, measurements on rippled membranes show a spontaneous magnetic moment at room temperature, with a saturation magnetization of 5.2 bohr magneton per $\mathrm{Gd}$. First-principles calculations show that the coupling to homogeneous strain is too small to induce ferromagnetism, suggesting a dominant role for strain gradients. Our membranes provide a novel platform for tuning the magnetic properties of intermetallic compounds via strain (piezomagnetism and magnetostriction) and strain gradients (flexomagnetism).

\footnotetext{
${ }^{1}$ Materials Science and Engineering, University of Wisconsin-Madison, Madison, WI, USA. ${ }^{2}$ Department of Physics and Astronomy, Rutgers University, New Brunswick, NJ, USA. ${ }^{凶}$ email: jkawasaki@wisc.edu
} 
$\mathrm{M}$ embranes are a powerful platform for flexible devices and for tuning properties via strain and strain gradients $^{1-7}$. In contrast to the uniform strain of epitaxial films, strain in membranes can be applied dynamically, anisotropically, in gradient form, and at larger magnitudes. For example, recent experiments on ultrathin oxide membranes demonstrate the application of extreme uniaxial strain of $8 \%^{1}$, whereas the maximum strain possible in an epitaxial thin film is typically no more than $3 \%$ before plastic deformation. In addition, membranes enable the application of strain gradients, which are difficult to control for a film that is rigidly clamped to a substrate (Fig. 1).

Magnetism is particularly attractive for tuning via strain in the membrane form. The coupling between magnetism and strain $\epsilon$, i.e., piezomagnetism $(M \propto \epsilon)$ and magnetostriction $\left(M^{2} \propto \epsilon\right)$, is widely used to tune the magnetic properties of thin films ${ }^{8-11}$. In contrast, the magnetic coupling to strain gradients, i.e., flexomagnetism $(M \propto \nabla \epsilon)$, has been theoretically predicted ${ }^{12,13}$, but to our knowledge has not experimentally demonstrated. This is due, in part, to difficulties in synthesizing and controlling strain gradients in nanostructures and membranes. Conventional techniques for fabricating single-crystalline membranes require etching of a sacrificial layer ${ }^{2}, 3,5,14$, which requires a detailed knowledge of etch chemistry and limits the ability to make ultrathin membranes of air-sensitive materials.

We demonstrate the etch-free epitaxial synthesis and exfoliation of Heusler membranes and show that rippled membranes induce magnetic ordering, turning the antiferromagnetic half Heusler compound GdPtSb into a ferro- or ferrimagnet. Heusler compounds are a broad class of intermetallic compounds with tunable magnetic textures ${ }^{15}$, topological states ${ }^{16-18}$, and novel superconductivity ${ }^{19,20}$. In contrast with conventional membrane synthesis techniques, which require etching of a sacrificial buffer layer ${ }^{1-4,14}$, our use of a monolayer graphene decoupling layer allows GdPtSb membranes to be mechanically exfoliated, bypassing the need for detailed knowledge of etch chemistries. Our approach is akin to "remote epitaxy," which has recently been demonstrated for the growth of compound semiconductor ${ }^{21,22}$, transition metal oxide ${ }^{7}$, halide perovskite ${ }^{23}$, and elemental metal films ${ }^{24}$. Here, we show that similar approaches apply to intermetallic quantum materials. We find that the large strains and strain gradients in rippled GdPtSb membranes drive an antiferromagnet to ferri- or ferromagnet transition. First-principles calculations show that the coupling to homogeneous strain is too small to induce ferromagnetism,

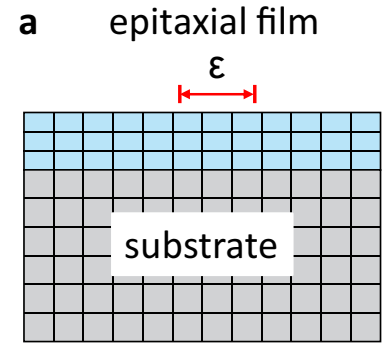

uniform strain $\varepsilon$

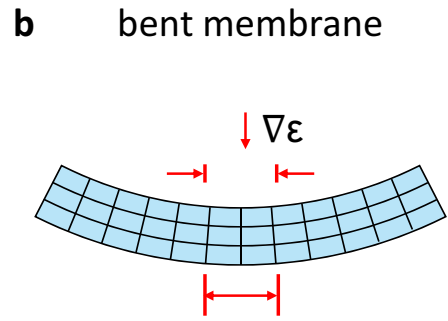

strain gradient $\nabla \varepsilon$
Fig. 1 Modes of strain accessible to epitaxial films vs free-standing membranes. a Piezomagnetism and magnetostriction are magnetic responses to uniform strain $(\epsilon)$ and are accessible in thin films. b Flexomagnetism is the response to strain gradients $(\nabla \epsilon)$ and is accessible in ultrathin membranes. suggesting a dominant role for strain gradients, which would make this system the first experimental example of flexomagnetic coupling. Our work opens a new platform for driving ferroic phase transitions via complex strained geometries.

\section{Results}

Epitaxy of GdPtSb on graphene-terminated sapphire. Our concept relies on the weak van der Waals interactions of monolayer graphene to enable epitaxial growth and exfoliation of a membrane from a graphene-terminated single-crystalline substrate. Figure 2a shows a schematic heterostructure, which consists of cubic GdPtSb (space group $\mathrm{F} \overline{4} 3 \mathrm{~m}$ ), polycrystalline monolayer graphene, and a single-crystalline $\mathrm{Al}_{2} \mathrm{O}_{3}$ substrate in (0001) orientation. GdPtSb crystallizes in the cubic half Heusler structure, the same structure as the antiferromagnetic Weyl semimetal $\mathrm{GdPtBi}{ }^{16,25,26}$. Since the layer-transferred graphene
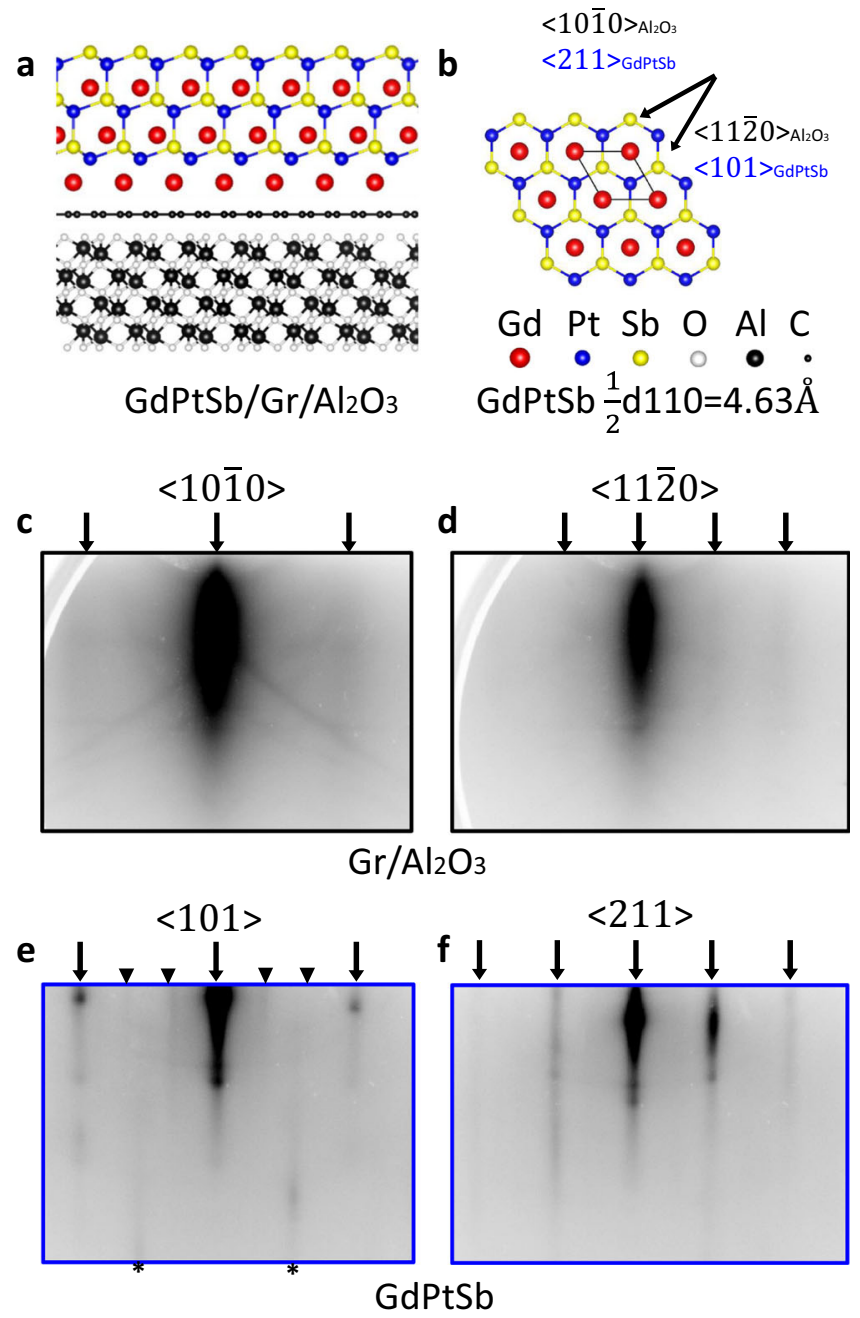

Fig. 2 Epitaxy of GdPtSb on graphene-terminated $\mathrm{Al}_{2} \mathrm{O}_{3}$ (0001). a Schematic crystal structure of (111)-oriented cubic GdPtSb on graphene/ $\mathrm{Al}_{2} \mathrm{O}_{3}$ (0001). The lattice parameter for $\mathrm{Al}_{2} \mathrm{O}_{3}$ is $a=4.785 \AA$ and that of GdPtSb is $\frac{1}{2} d_{110}=4.53 \AA$, corresponding to a mismatch of $2.7 \%$. b In-plane crystal structure of GdPtSb (111). c, d Reflection high-energy electron diffraction (RHEED) patterns of graphene on $\mathrm{Al}_{2} \mathrm{O}_{3}$ after a $700^{\circ} \mathrm{C}$ annealing. Black arrows mark the underlying $\mathrm{Al}_{2} \mathrm{O}_{3}$ reflections. e, f RHEED patterns for the GdPtSb film. Black arrows mark the bulk reflections. Triangles mark superstructure reflections from a $3 \times$ surface reconstruction. Additional reflections (black asterisks) are observed that correspond to a second domain rotated by $\pm 30^{\circ}$. 
has randomly oriented polycrystalline domains, if the primary interactions are between GdPtSb film and graphene, then a polycrystalline film is expected. If, on the other hand, the primary interactions are between GdPtSb film and the underlying substrate, then an epitaxial film is expected. Given the recent demonstration of semi-lattice transparency of graphene during GaAs/graphene/GaAs "remote epitaxy"21, we expect epitaxial registry between GdPtSb and the underlying sapphire to dominate. Single-crystalline GdPtSb membranes can then be exfoliated and strained in complex geometries.

To synthesize the GdPtSb/graphene/ $\mathrm{Al}_{2} \mathrm{O}_{3}$ (0001) heterostructures, we first transfer polycrystalline monolayer graphene onto a pre-annealed $\mathrm{Al}_{2} \mathrm{O}_{3}$ (0001) substrate using standard wet transfer techniques ("Methods"). The graphene is grown on copper foil by chemical vapor deposition. Raman spectroscopy and atomic force microscopy indicate clean transfers with long-range coverage and minimal point defects or tears in the graphene (Supplementary Fig. S1). We then anneal the graphene/sapphire samples at $700{ }^{\circ} \mathrm{C}$ in ultrahigh vacuum $\left(P<2 \times 10^{-10}\right.$ Torr $)$ to clean the surface. At this stage, the reflection high-energy electron diffraction (RHEED) pattern shows a bright but diffuse specular reflection compared to bare sapphire ${ }^{27}$, which we attribute to diffuse scattering from the randomly oriented top graphene layer (Fig. 2c, d). There are weak diffraction streaks at the +1 and -1 positions (arrows), which we attribute to the underlying sapphire substrate.

GdPtSb films were grown by molecular beam epitaxy (MBE) on the graphene $/ \mathrm{Al}_{2} \mathrm{O}_{3}$ at $600^{\circ} \mathrm{C}$, using conditions similar to ref. ${ }^{27}$ ("Methods"). The streaky RHEED patterns for GdPtSb indicate growth with the epitaxial registry to the underlying sapphire substrate (Fig. 2e, f, black arrows). For beam oriented along $\langle 101\rangle_{\mathrm{GdPtSb}}$, we also observe superstructure reflections corresponding to a $3 \times$ surface reconstruction (Fig. 2e, triangles), indicating a well-ordered surface. In addition to the expected streaks for a hexagon-on-hexagon epitaxial relationship (black arrows), we observe faint secondary streaks marked by asterisks. The $\Delta Q$ spacing between these streaks differs from the main reflections (arrows) by a factor of $\sqrt{3}$, suggesting the presence of two domains that are rotated by $30^{\circ}$ : one domain with the expected $\langle 101\rangle_{\mathrm{GdPtSb}} \|\langle 11 \overline{2} 0\rangle_{\mathrm{Al}_{2} \mathrm{O}_{3}}$ epitaxial relationship, and the other rotated by $\pm 30^{\circ}$ around the $\mathrm{Al}_{2} \mathrm{O}_{3}$ [0001] axis with the epitaxial relationship $\langle 101\rangle_{\mathrm{GdPtSb}} \|\langle 10 \overline{1} 0\rangle_{\mathrm{Al}_{2} \mathrm{O}_{3}}$.

XRD pole figure and cross-sectional TEM measurements confirm the presence of these two epitaxial domains. Figure $3 \mathrm{c}$ shows a pole figure of the GdPtSb 220 and $\mathrm{Al}_{2} \mathrm{O}_{3} 10 \overline{1} 4$ reflections. We observe two sets of domains, one corresponding to the expected $\langle 101\rangle_{\mathrm{GdPtSb}} \|\langle 11 \overline{2} 0\rangle_{\mathrm{Al}_{2} \mathrm{O}_{3}}$ relationship, and the other set rotated by $\pm 30^{\circ}$. In contrast, GdPtSb films grown directly on sapphire do not show the $\pm 30^{\circ}$ domains (Supplementary Fig. S2). We speculate that the second domain forms for heterostructures with graphene because the weak interactions across the graphene change the balance between the energy of interfacial bonding and the strain energy, favoring small strains via a lattice rotation. A $0^{\circ}$ GdPtSb domain has a lattice mismatch with a sapphire of $2.7 \%$, whereas a $\pm 30^{\circ}$ rotation corresponds to a $(3,3)_{\mathrm{GdPtSb}} \|(5,0)_{\mathrm{Al}_{2} \mathrm{O}_{3}}$ superstructure with a mismatch of only $-1.5 \%$. Here, we write the GdPtSb lattice vectors in hexagonal coordinates, where ( $\mathbf{a}^{\prime}|| 10 \overline{1}, \mathbf{b}^{\prime}|| 11 \overline{1} 0$ ). Rotational domains have also been observed for $\mathrm{GaN}$ films grown on monolayer h-BN/GaN $(0001)^{22}$ and for $\mathrm{Cu}$ films growth on monolayer graphene $/ \mathrm{Al}_{2} \mathrm{O}_{3}(0001)^{24}$. However, in those cases, the presence of the second domain was attributed to an epitaxial relationship between the film $(\mathrm{Cu}$ or $\mathrm{GaN}$ ) and the $2 \mathrm{D}$ monolayer (h-BN or graphene), while the primary domain results from an epitaxial relationship between the film and the substrate. In the present case of $\pm 30^{\circ}$ domains of $\mathrm{GdPtSb}$, we rule out a long-range epitaxial relationship to the
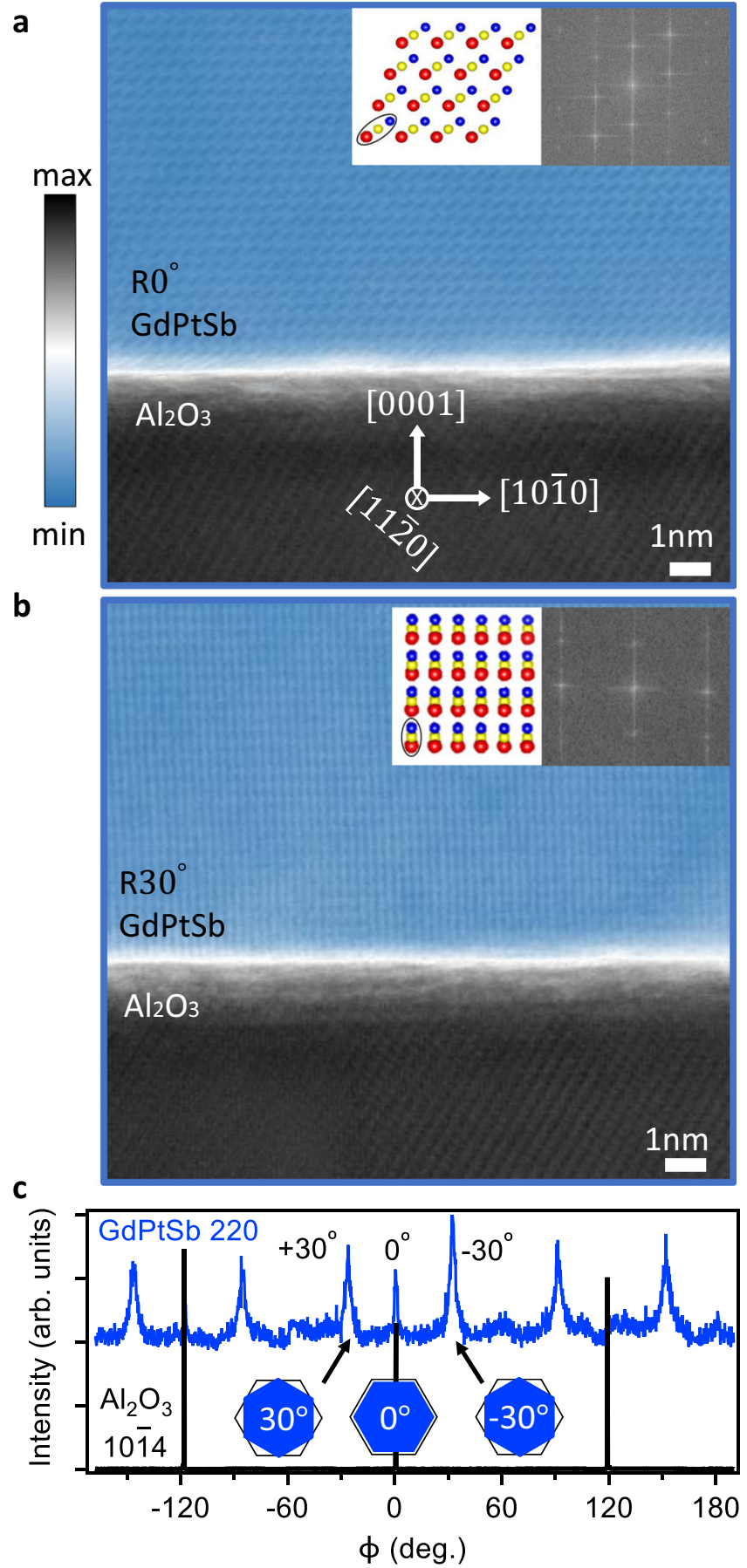

Fig. 3 Heusler/graphene/sapphire interface. a, b STEM image of the $\mathrm{GdPtSb} /$ graphene $/ \mathrm{Al}_{2} \mathrm{O}_{3}$ interface for the two different $\mathrm{GdPtSb}$ domains, as viewed along the $\langle 11 \overline{2} \mathrm{O}\rangle_{\mathrm{Al}_{2} \mathrm{O}_{3}}$ zone axis. We used log scale false-color image to simultaneously visualize the film and substrate for better contrast. The insets show the schematic crystal structures and fast Fourier transforms of the STEM images. c In-plane $\phi$ scan of GdPtSb 220 reflections and $\mathrm{Al}_{2} \mathrm{O}_{3} 10 \overline{1} 4$ reflections for $\mathrm{GdPtSb}$ sample grown on graphene $/ \mathrm{Al}_{2} \mathrm{O}_{3} . \Delta \phi= \pm 30^{\circ}$ are the in-plane angles between the $\langle 101\rangle$ direction of GdPtSb and the $\langle 11 \overline{2} \mathrm{O}\rangle$ direction of $\mathrm{Al}_{2} \mathrm{O}_{3}$ substrate.

graphene because our graphene barrier is polycrystalline, and thus if there were an epitaxial relationship between GdPtSb and polycrystalline graphene, a large distribution of GdPtSb domain orientations would result.

Annular bright-field (ABF) scanning transmission electron microscopy (STEM) images in Fig. 3a, b confirm the existence of 
these two sets of domains. In these images, the atomic structure is resolved a few nanometers away from the interface. It is difficult to resolve the registry at the GdPtSb/graphene/sapphire interface, which we attribute to partial film delamination during TEM sample preparation. The STEM image of the $\Delta \phi=0$ domain consists of arrays of tilted spindle-shaped dark spots, each of which represents a combination of one $\mathrm{Gd}$ atomic column, one $\mathrm{Pt}$ atomic column, and one $\mathrm{Sb}$ atomic column. For the STEM image of the $\pm 30^{\circ}$ rotated $\mathrm{GdPtSb}$, the spindle-shaped spots are aligned along the vertical direction corresponds to clusters of Gd-Pt-Sb atomic columns, as shown by the inset cartoon.

Membrane exfoliation. Heusler films grown on graphene $/ \mathrm{Al}_{2} \mathrm{O}_{3}$ can be mechanically exfoliated to yield free-standing membranes, without the need for a metal stressor release layer. Figure $4 \mathrm{a}$ shows $\theta-2 \theta$ scans of GdPtSb, before and after exfoliation. Before exfoliation, we observe all of the expected 111, 222, 333, and 444 reflections. The rocking curve width of the 111 reflections before exfoliation is 15.4 arc second, indicating a high-quality film (Supplementary Fig. S3). After mechanical exfoliation (blue curve), we observe all of the expected GdPtSb reflections and none from the substrate, indicating a high crystallographic quality of the exfoliated membranes.

We find that the best exfoliation with minimal cracks is performed by adhering the sample film-side down to a rigid glass slide using Crystalbond and then prying off the substrate. The resulting membranes adhered to the glass slide have minimal long-range tears, as shown in Fig. 4b and Supplementary Fig. S5. It is also possible to exfoliate by adhering Kapton or thermal release tape directly to the film and peeling off the membrane; however, the bending during this peeling process produces microtears (Supplementary Fig. S4). An important aspect of this system is that unlike the compound semiconductor ${ }^{21}$ or oxide 28 systems grown by "remote epitaxy," in this Heusler/graphene/ $\mathrm{Al}_{2} \mathrm{O}_{3}$ system, no metal stressor layer was required in order to perform the exfoliation. As a control, GdPtSb films grown directly on sapphire could not be exfoliated by these methods.

Strain-induced magnetism in rippled membranes. We now demonstrate that the strains and/or large strain gradients in rippled Heusler membranes induce magnetic ordering, transforming antiferromagnetic GdPtSb films into ferro- (or ferri-) magnetic rippled membranes at room temperature. Figure 5a shows the magnetization $M$ versus applied magnetic field $H$ for a relaxed epitaxial GdPtSb film on $\mathrm{Al}_{2} \mathrm{O}_{3}$ (green) and for rippled membranes on two different polymer handles (blue and red). The unstrained film shows a weak linear $M(H)$ dependence. Temperature-dependent susceptibility measurements indicate that the films become antiferromagnetic at a Néel temperature of $\sim 12 \mathrm{~K}$ (Supplementary Fig. S7). This behavior is consistent with the closely related $\mathrm{GdPtBi}$, a $G$-type antiferromagnet $\left(T_{N} \sim 8.5 \mathrm{~K}\right)$ in which local Gd moments are aligned ferromagnetically within (111) planes and the planes are aligned antiferromagnetically with neighboring planes ${ }^{29}$. Our first-principles calculations are consistent with an antiferromagnetic ground state for GdPtSb (Supplementary Fig. S9).

In contrast, rippled GdPtSb membranes on polyimide (blue) and polyurethane (red) layers show a spontaneous magnetization and hysteresis loops characteristic of ferrimagnetic (FiM) or ferromagnetic (FM) order. The saturation magnetization in the most rippled membrane on polyurethane is $5.2 \mu_{B}$ per Gd atom, approaching the $\sim 7 \mu_{B} / \mathrm{Gd}$ ferromagnetic limit (Fig. 5a). We observe a systematic dependence of the saturation magnetization (and magnetic susceptibility) on the ripple aspect ratio (height/width, Fig. $5 c-f)$, suggesting that the origins of the magnetic order are
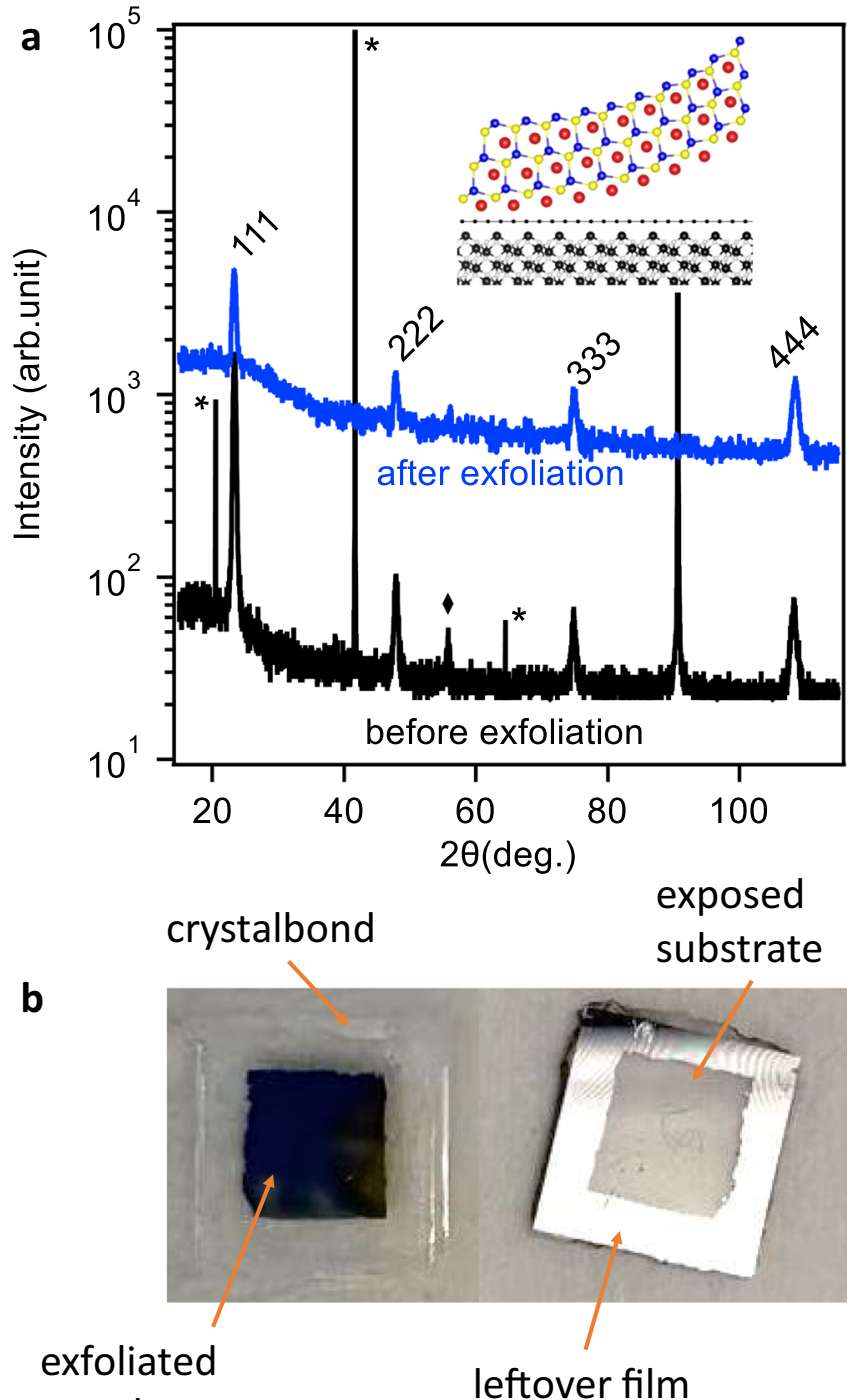

\section{membrane}

Fig. 4 Crystallography and exfoliation of flat GdPtSb membranes. a XRD $\theta-2 \theta$ ( $\mathrm{Cu} K \alpha$ ) scans before exfoliation (black) and after exfoliation (blue). Sapphire substrate reflections are marked by asterisks. In addition to the epitaxial III-type reflections, a small 004 reflection is observed and marked with a diamond. $\mathbf{b}$ Photos of GdPtSb membrane exfoliation from a $1 \times 1 \mathrm{~cm}^{2}$ substrate. The left image shows a flat exfoliated membrane bonded to a glass slide. The right image shows the substrate side after exfoliation. In the region that had been covered with graphene $\left(\sim 6 \times 6 \mathrm{~mm}^{2}\right.$ square at center), the GdPtSb membrane has been exfoliated to yield a transparent sapphire substrate. In the region not covered by graphene, the GdPtSb film remains adhered to the substrate.

ripple-induced strain or strain gradients, as opposed to extrinsic effects. These rippled membranes are made by adhering the membrane side of a flat membrane/crystalbond/glass slide stack to polymers with different thermal expansion coefficients (polyimide $\alpha=34 \times 10^{-6} \mathrm{~K}^{-1}$ or polyurethane $\left.\alpha=57 \times 10^{-6} \mathrm{~K}^{-1}\right)$. Melting the crystalbond on a hot plate releases the membrane/polymer bilayer. Ripples form upon release and cooling (Supplementary Fig. S6). We find that membranes on polymers with larger thermal expansion coefficients have larger ripple heights.

Our observation of ripple-induced magnetism in Heusler lies in contrast with previous studies of strain coupling in magnetic Heusler compounds. Previous studies have focused on the strain tuning of magnetic anisotropy, either in epitaxial films ${ }^{30}$ or by 
a

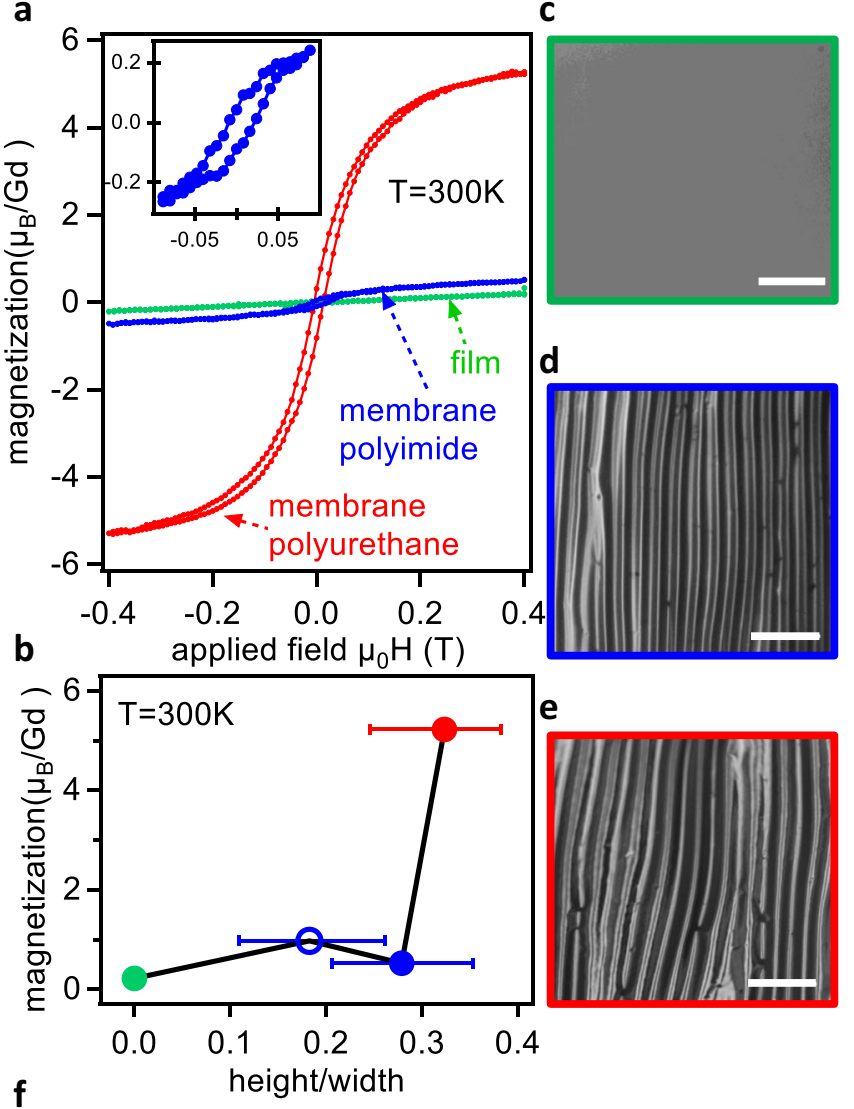

f

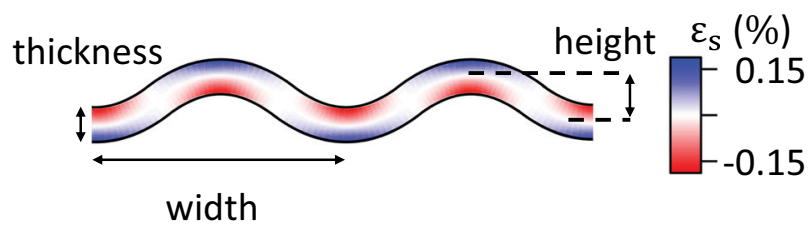

Fig. 5 Strain-induced magnetism in rippled GdPtSb membranes. a

Magnetization of GdPtSb thin film directly grown on a sapphire substrate (green), exfoliated GdPtSb membrane adhered to polyimide (blue) and polyurethane (red). The applied field $H$ is oriented out-of-the sample plane, i.e., the global [111] direction. Both membranes have a thickness of $14 \mathrm{~nm}$. b Room-temperature magnetization at 0.4 Tesla versus ripple aspect ratio (height/width). Relaxed film (green), rippled membrane on polyimide (filled and open blue circles), rippled membrane on polyurethane (red). The ripple height and width are measured by phase-contrast optical microscopy. The error bar represents the distribution of aspect ratio over several regions of the rippled membranes. c-e Optical microscope images of the film c, rippled membrane on polyimide $\mathbf{d}$, and rippled membrane on polyurethane $\mathbf{e}$, respectively. The scale bar is $100 \mu \mathrm{m}$. $\mathbf{f}$ Estimate of the strain $\epsilon_{s}$ for a sinusoidal membrane with peak to peak width (wavelength) 20 micron, ridge to valley height 5 micron, and thickness $14 \mathrm{~nm}$, consistent with the dimensions of the membrane on polyurethane. Details in Supplementary Fig. S8.

bending in polycrystalline membranes on a flexible substrate ${ }^{31}$. In this study, we observe not a tunable anisotropy, but a straininduced magnetic ordering. Other studies have investigated magnetoelastic coupling in ferromagnetic shape memory alloys $^{32,33}$, where the magnetic phase transition is coupled with a structural martensitic phase transition.

\section{Discussion}

Due to the short-range nature of exchange interactions, subtle changes in bond length and local symmetry are expected to modify the magnetic ground state, consistent with our observed AFM to FM (FiM) transition. For example, long-range and oscillatory Ruderman-Kittel-Kasuya-Yosida (RKKY) interactions were detected in some Mn-based Heuslers ${ }^{34,35}$. The RKKY interaction oscillates in sign and magnitude with the distance between magnetic atoms, exhibiting oscillation between ferromagnetic (FM) and antiferromagnetic (AFM) ordering ${ }^{36-38}$. However, the atomic-scale mechanism for ripple-induced magnetism in GdPtSb is not fully understood. A key question is whether the AFM to ferro- or ferrimagnetic ordering in our rippled membranes is driven by strain (piezomagnetism and magnetostriction) or strain gradients (flexomagnetism). Both strain and strain gradients are present in our rippled membranes, and both increase with the ripple aspect ratio. While piezomagnetism and magnetostriction have been widely studied, to our knowledge flexomagnetism has only been predicted $^{12,13}$, but not experimentally observed.

To estimate the relative contributions of strain and strain gradients, in Fig. $5 f$, we estimate the strain for a sinusoidal ripple in a membrane with the same thickness, height, and width as the rippled GdPtSb membrane on polyurethane. The magnitudes of strain $\epsilon_{s}$ along the sinusoidal paths are modest, with peak values of $\pm 0.17 \%$. In our first-principles calculations, $\pm 0.17 \%$ is much too small a strain to induce a ferromagnetic or ferrimagnetic state, with a much larger strain of $\sim 5 \%$ being required (Supplementary Fig. S9). In contrast, the estimated out-of-plane strain gradients $\mathrm{d} \epsilon_{s} / \mathrm{d} t$ are large, with peak values $\pm 25 \%$ per micron (Supplementary Fig. S8). These large differences in magnitude suggest that the gradient term may dominate and that the observed behavior is flexomagnetic coupling; however, direct measurements of the strain state and direct calculations of the flexomagnetic response are required to fully understand the origins of magnetization. Our membranes provide a platform for the control and detailed understanding of the coupling between strain, strain gradients, and magnetism.

Our work also has strong implications for the remote epitaxial growth and other strain-induced properties in single-crystalline membranes. First, we expand the range of new functional materials that can be grown on graphene, to include intermetallic systems with mixed covalent and metallic bonding. Previous demonstrations of remote epitaxy have focused on transition metal oxides ${ }^{28}$, halide perovskites ${ }^{23}$, and compound semiconductors ${ }^{21}$, which have more ionic bonding character. Second, strain and strain gradients in rippled membranes provide a platform for tuning other materials properties and ferroic orders, beyond magnetism. For example, it is an outstanding challenge to electrically switch the polarization of a polar metal due to strong charge screening. First-principals calculations suggest that strain gradients could be used to switch a polar metal via flexoelectric coupling ${ }^{39}$, analogous to the possible flexomagnetism investigated here.

\section{Methods}

Synthesis of graphene. Graphene was grown using thermal chemical vapor deposition of ultra-high purity $\mathrm{CH}_{4}$ at $1050{ }^{\circ} \mathrm{C}$ on $\mathrm{Cu}$ foil. As-received copper foils (BeanTown Chemical number 145780, 99.8\% purity) were cut into 1-inch-by-1inch pieces and soaked in dilute nitric acid (5.7\%) for $40 \mathrm{~s}$ followed by $3 \times$ DI water rinse followed by soaking in acetone and IPA to remove water from the surface. Dilute nitric acid helps remove the oxide and impurity particles from the surface. Foils were then dried under a gentle stream of air. Foils were subsequently loaded into a horizontal quartz tube furnace in which the furnace can slide over the length of the tube. Prior to monolayer graphene synthesis, the CVD chamber was evacuated to $<10^{-2}$ Torr using a scroll pump. The system was then back-filled with Ar and $\mathrm{H}_{2}$, and a steady flow (331 sccm Ar, $9 \mathrm{sccm} \mathrm{H}_{2}$ ) monitored by mass flow controllers was maintained at ambient pressure. The furnace was then slid to surround the samples, and annealed for $30 \mathrm{~min}$. Then $0.3 \mathrm{sccm}$ of $\mathrm{P}-5$ gas $\left(5 \% \mathrm{CH}_{4}\right.$ in Ar) was flowed for 45 min so that a monolayer of graphene formed on the surface. To terminate the growth, the furnace was slid away from the samples, and 
the portion of the quartz tube containing the samples was cooled to room temperature.

Transfer of graphene to $\mathbf{A l}_{\mathbf{2}} \mathbf{O}_{\mathbf{3}}$. Our graphene transfer procedure is a modified polymer-assisted wet transfer, similar to the transfer recipes studied in previous works ${ }^{40}$. (0001)-oriented $\mathrm{Al}_{2} \mathrm{O}_{3}$ substrates were prepared by annealing at $1400{ }^{\circ} \mathrm{C}$ for $10 \mathrm{~h}$ at atmospheric pressure in order to obtain a smooth, terrace-step morphology. To perform the graphene transfer, the graphene/Cu foils were cut into 6$\mathrm{mm}$-by-6-mm pieces and flattened using clean glass slides. Approximately $300 \mathrm{~nm}$ of 495K C2 PMMA (Chlorobenzene base solvent, 2\% by wt., MicroChem) was spin-coated on the graphene/Cu foil substrate at $2000 \mathrm{RPM}$ for $2 \mathrm{~min}$ and left to cure at room temperature for $24 \mathrm{~h}$. Graphene on the backside of the $\mathrm{Cu}$ foil was removed via reactive ion etching using $90 \mathrm{~W} \mathrm{O}_{2}$ plasma at a pressure of $100 \mathrm{mTorr}$ for $30 \mathrm{~s}$. The $\mathrm{Cu}$ foil was then etched by placing the PMMA/graphene/Cu foil on the surface of an etch solution containing 1-part ammonium persulfate (APS-100, Transene) and 3-parts $\mathrm{H}_{2} \mathrm{O}$. After $10 \mathrm{~h}$ of etching at room temperature, the floating PMMA/graphene membrane was scooped up with a clean glass slide and sequentially transferred into three 30 -min water baths to rinse the etch residuals. The PMMA/graphene membrane was then scooped out of the final water bath using the annealed sapphire substrate, to yield a PMMA/graphene/ $\mathrm{Al}_{2} \mathrm{O}_{3}$ stack.

To remove water at the graphene $/ \mathrm{Al}_{2} \mathrm{O}_{3}$ interface, samples were baked in the air at $50^{\circ} \mathrm{C}$ for $5 \mathrm{~min}$, then slowly ramped to $150{ }^{\circ} \mathrm{C}$ and baked for another $10 \mathrm{~min}$. The PMMA is removed by submerging the sample in an acetone bath at $80^{\circ} \mathrm{C}$ for $3 \mathrm{~h}$. This is followed by an isopropanol and water rinse. The sample is indium bonded onto a molybdenum puck and outgassed at $150{ }^{\circ} \mathrm{C}$ for $1 \mathrm{~h}$ in a loadloack at a pressure $P<5 \times 10^{-7}$ Torr before introduction to the MBE growth chamber. Finally, the graphene $/ \mathrm{Al}_{2} \mathrm{O}_{3}$ sample is annealed at $400^{\circ} \mathrm{C}$ for $1 \mathrm{~h}$ in the MBE chamber to desorb remaining organic residues and then annealed up to $700{ }^{\circ} \mathrm{C}$ immediately prior to the growth of GdPtSb.

Molecular beam epitaxy of GdPtSb films. GdPtSb films were grown on graphene $/ \mathrm{Al}_{2} \mathrm{O}_{3}$ by molecular beam epitaxy $(\mathrm{MBE})$ at a sample temperature of $600{ }^{\circ} \mathrm{C}$, using conditions similar to ref. ${ }^{27}$. Gd flux was supplied by a thermal effusion cell. A mixture of $\mathrm{Sb}_{2}$ and $\mathrm{Sb}_{1}$ was supplied by a thermal cracker cell with a cracker zone operated at $1200^{\circ} \mathrm{C}$. The Pt flux was supplied by an electron beam evaporator. Fluxes were measured in situ using a quartz crystal microbalance (QCM) and calibrated with Rutherford Backscattering Spectroscopy. The Gd to Pt atomic flux ratio was maintained to be $1: 1$. Due to the high relative volatility of $\mathrm{Sb}, \mathrm{GdPtSb}$ films were grown in an Sb adsorption-controlled regime with a $30 \%$ excess Sb flux. After the growth, films were capped in situ with $75 \mathrm{~nm}$ amorphous $\mathrm{Ge}$ to protect the surfaces from oxidation.

Raman and atomic force microscopy of graphene/ $\mathbf{A l} \mathbf{O}_{\mathbf{3}}$. Graphene transfer coverage and cleanliness is studied using field emission scanning electron microscopy (SEM) (Zeiss LEO 1530 Gemini). The graphene quality after the transfer is assessed via Raman spectroscopy using a $532 \mathrm{~nm}$ wavelength laser (Thermo Scientific DXR Raman Microscope). The laser power is kept below $5 \mathrm{~mW}$ in order to prevent damage to the graphene. The terrace-step morphology of the annealed sapphire substrates with and without graphene termination is analyzed by AFM (Bruker Multimode 8 SPM) in tapping mode.

Scanning transmission electron microscopy. The $\mathrm{GdPtSb} /$ graphene/ $\mathrm{Al}_{2} \mathrm{O}_{3}$ sample was prepared for TEM using a Zeiss Auriga focused ion beam (FIB) with a final FIB polishing step with a $5 \mathrm{kV} 100 \mathrm{pA}$ Ga-ion beam. The sample surface was further polished for a higher smoothness in a Fishione 1040 Nanomill with a 900 $\mathrm{eV}$ Ar ion beam, to a thickness of $\sim 80 \mathrm{~nm}$. We did not seek to get the thinnest possible sample, as the film layer could peel off from the substrate when the sample is too thin. The TEM sample was kept under vacuum and cleaned in an Ibss GV10x DS Asher plasma cleaner for $10 \mathrm{~min}$ under $20 \mathrm{~W}$ to remove contaminations before being inserted into the TEM column.

A Thermo-Fisher Titan STEM equipped with a CEOS probe aberration corrector operated at $200 \mathrm{kV}$ was used to collect STEM data. A $24.5 \mathrm{mrad}$ semi convergence angle and an $18.9 \mathrm{pA}$ current probe were used. A Gatan BF/ABF detector with $5.7 \mathrm{mrad}$ and $22.8 \mathrm{mrad}$ inner and outer collection angles was used to collect the annular bright-field (ABF) images.

SQUID measurements. Magnetic properties were measured using a Quantum Design MPMS SQUID (Superconducting Quantum Interference Device) Magnetometer. For the film data, we subtract a background measurement of the $\mathrm{Al}_{2} \mathrm{O}_{3}$ substrate. For the membrane samples, we subtract a background measurement of the polymer tape (polyimide or polyurethane) + Crystalbond.

Density-functional theory calculations. Density-functional theory (DFT) calculations were carried out with the ABINIT package. The pseudopotentials used were PAW JTH v1.1 ${ }^{41}$ within the local density approximation. Calculations were done on a conventional unit cell (simple cubic with four formula units per cell) with $6 \times$ $6 \times 6$ Monkhorst-Pak k-point mesh, a plane-wave cutoff of 25 Hartree, with spin-orbit coupling included. The criterion for convergence was a potential residual of $<1.0 \times 10^{-8}$.

\section{Data availability}

All data and code used in this paper will be made available upon reasonable request.

Received: 17 June 2020; Accepted: 29 March 2021;

Published online: 03 May 2021

\section{References}

1. Hong, S. S. et al. Extreme tensile strain states in $\mathrm{La}_{0.7} \mathrm{Ca}_{0.3} \mathrm{MnO}_{3}$ membranes Science 368, 71-76 (2020).

2. Halliday, D., Eggleston, J., Lee, K., Frost, J. \& Beaumont, S. Optical properties of ultrathin 50nm GaAs membranes. Solid State Commun. 96, 359-365 (1995).

3. Fang, D., Striemer, C., Gaborski, T., McGrath, J. \& Fauchet, P. Methods for controlling the pore properties of ultra-thin nanocrystalline silicon membranes. J. Phys.: Condens. Matter 22, 454134 (2010).

4. Snyder, J. et al. An experimental and theoretical analysis of molecular separations by diffusion through ultrathin nanoporous membranes. J. Membr. Sci. 369, 119-129 (2011).

5. Roberts, M. M. et al. Elastically relaxed free-standing strained-silicon nanomembranes. Nat. Mater. 5, 388-393 (2006).

6. Levy, N. et al. Strain-induced pseudo-magnetic fields greater than 300 Tesla in graphene nanobubbles. Science 329, 544-547 (2010).

7. Ji, D. et al. Freestanding crystalline oxide perovskites down to the monolayer limit. Nature 570, 87-90 (2019).

8. Lee, E. W. Magnetostriction and magnetomechanical effects. Rep. Progr. Phys 18, 184 (1955).

9. Callen, E. \& Callen, H. B. Magnetostriction, forced magnetostriction, and anomalous thermal expansion in ferromagnets. Phys. Rev. 139, A455 (1965).

10. Sander, D. et al. Stress, strain and magnetostriction in epitaxial films. J. Phys.: Condens. Matter 14, 4165 (2002).

11. Weber, M., Koch, R. \& Rieder, K. Uhv cantilever beam technique for quantitative measurements of magnetization, magnetostriction, and intrinsic stress of ultrathin magnetic films. Phys. Rev. Lett. 73, 1166 (1994).

12. Lukashev, P. \& Sabirianov, R. F. Flexomagnetic effect in frustrated triangular magnetic structures. Phys. Rev. B 82, 094417 (2010).

13. Eliseev, E. A., Morozovska, A. N., Glinchuk, M. D. \& Blinc, R. Spontaneous flexoelectric/flexomagnetic effect in nanoferroics. Phys. Rev. B 79, 165433 (2009).

14. Lu, D. et al. Synthesis of freestanding single-crystal perovskite films and heterostructures by etching of sacrificial water-soluble layers. Nat. Mater. 15, 1255-1260 (2016)

15. Nayak, A. K. et al. Magnetic antiskyrmions above room temperature in tetragonal heusler materials. Nature 548, 561-566 (2017).

16. Hirschberger, M. et al. The chiral anomaly and thermopower of weyl fermions in the half-Heusler GdPtBi. Nat. Mater. 15, 1161-1165 (2016).

17. Logan, J. A. et al. Observation of a topologically non-trivial surface state in half-heusler PtLuSb (001) thin films. Nat. Commun. 7, 1-7 (2016).

18. Liu, Z. et al. Observation of unusual topological surface states in half-heusler compounds LnPtBi. Nat. Commun. 7, 12924 (2016).

19. Kim, H. et al. Beyond triplet: unconventional superconductivity in a spin-3/2 topological semimetal. Sci. Adv. 4, eaao4513 (2018).

20. Brydon, P., Wang, L., Weinert, M. \& Agterberg, D. Pairing of $j=3 / 2$ fermions in half-heusler superconductors. Phys. Rev. Lett. 116, 177001 (2016).

21. Kim, Y. et al. Remote epitaxy through graphene enables two-dimensional material-based layer transfer. Nature 544, 340-343 (2017).

22. Kong, W. et al. Polarity governs atomic interaction through two-dimensional materials. Nat. Mater. 17, 999-1004 (2018).

23. Jiang, J. et al. Carrier lifetime enhancement in halide perovskite via remote epitaxy. Nat. Commun. 10, 1-12 (2019).

24. Lu, Z. et al. Remote epitaxy of copper on sapphire through monolayer graphene buffer. Nanotechnology 29, 445702 (2018).

25. Suzuki, T. et al. Large anomalous hall effect in a half-Heusler antiferromagnet. Nat. Phys. 12, 1119-1123 (2016).

26. Shekhar, C. et al. Anomalous hall effect in weyl semimetal half-heusler compounds RPtBi (R=Gd and Nd). Proc. Natl Acad. Sci. USA 115, 9140-9144 (2018).

27. Du, D. et al. High electrical conductivity in the epitaxial polar metals LaAuGe and LaPtSb. APL Mater. 7, 121107 (2019).

28. Kum, H. S. et al. Heterogeneous integration of single-crystalline complexoxide membranes. Nature 578, 75-81 (2020).

29. Kreyssig, A. et al. Magnetic order in GdPtBi studied by x-ray resonant magnetic scattering. Phys. Rev. B 84, 220408 (2011). 
30. Pechan, M. J., Yu, C., Carr, D. \& Palmstrøm, C. J. Remarkable strain-induced magnetic anisotropy in epitaxial $\mathrm{Co}_{2} \mathrm{MnGa}\left(\begin{array}{lll}0 & 0 & 1\end{array}\right)$ films. J. Magn. Magn. Mater. 286, 340-345 (2005).

31. Gueye, M. et al. Bending strain-tunable magnetic anisotropy in co2feal heusler thin film on kapton ${ }^{\circledR}$. Appl. Phys. Lett. 105, 062409 (2014).

32. Xuan, H. et al. Large magnetoresistance and magnetic field induced strain in $\mathrm{Ni}_{42.8} \mathrm{Co}_{7.7} \mathrm{Mn}_{38.8} \mathrm{Al}_{10.7}$ Heusler alloy at room temperature. Physica Status Solidi 215, 1800185 (2018).

33. Fujita, A., Fukamichi, K., Gejima, F., Kainuma, R. \& Ishida, K. Magnetic properties and large magnetic-field-induced strains in off-stoichiometric Ni-Mn-Al Heusler alloys. Appl. Phys. Lett. 77, 3054-3056 (2000).

34. Noda, Y. \& Ishikawa, Y. Spin waves in heusler alloys $\mathrm{Pd}_{2} \mathrm{MnSn}$ and $\mathrm{Ni}_{2} \mathrm{MnSn}$. J. Phys. Soc. Japan 40, 690-698 (1976).

35. Tajima, K. et al. Spin waves in a Heusler alloy $\mathrm{Cu}_{2} \mathrm{MnAl}$. J. Phys. Soc. Japan 43, 483-489 (1977).

36. Şaşığlu, E., Sandratskii, L. \& Bruno, P. Role of conduction electrons in mediating exchange interactions in Mn-based Heusler alloys. Phys. Rev. B 77, 064417 (2008).

37. Şaşığlu, E., Sandratskii, L. \& Bruno, P. Magnetic phase diagram of the semiHeusler alloys from first principles. Appl. Phys. Lett. 89, 222508 (2006).

38. Şaşığlu, E., Sandratskii, L., Bruno, P. \& Galanakis, I. Exchange interactions and temperature dependence of magnetization in half-metallic Heusler alloys. Phys. Rev. B 72, 184415 (2005)

39. Zabalo, A. \& Stengel, M. Switching a polar metal via strain gradients. Preprint at https://journals.aps.org/prl/abstract/10.1103/PhysRevLett.126.127601 (2020).

40. Park, H. et al. Optimized poly (methyl methacrylate)-mediated graphenetransfer process for fabrication of high-quality graphene layer. Nanotechnology 29, 415303 (2018).

41. Jollet, F., Torrent, M. \& Holzwarth, N. Generation of projector augmentedwave atomic data: A 71 element validated table in the xml format. Comput. Phys. Commun. 185, 1246-1254 (2014).

\section{Acknowledgements}

Heusler epitaxial film growth and graphene transfers were supported by the Army Research Office (ARO Award number W911NF-17-1-0254) and the National Science Foundation (DMR-1752797). TEM experiments by C.Z. and P.M.V. were supported by the US Department of Energy, Basic Energy Sciences (DE-FG02-08ER46547), and used facilities are supported by the Wisconsin MRSEC (DMR-1720415). Graphene synthesis and characterization are supported by the U.S. Department of Energy, Office of Science, Basic Energy Sciences, under award no. DE-SC0016007. DFT calculations by K.T.G. and K.M.S. were supported by the Office of Naval Research award number ONR N00014-191-2073. We gratefully acknowledge the use of X-ray diffraction facilities supported by the
NSF through the University of Wisconsin Materials Research Science and Engineering Center under Grant No. DMR-1720415.

\section{Author contributions}

D.D. and J.K.K. conceived the project. D.D. performed the Heusler film growth, diffraction, and magnetic measurements. S.M. performed the graphene transfer and characterization. V.S. and M.S.A. synthesized the graphene. C.Z. and P.M.V. performed TEM measurements. K.T.G. and K.M.R. performed DFT calculations. J.K.K. supervised the project. D.D. and J.K.K. wrote the paper with feedback from all authors.

\section{Competing interests}

The authors declare no competing interests.

\section{Additional information}

Supplementary information The online version contains supplementary material available at https://doi.org/10.1038/s41467-021-22784-y.

Correspondence and requests for materials should be addressed to J.K.K.

Peer review information Nature Communications thanks the anonymous reviewers for their contributions to the peer review of this work. Peer review reports are available.

Reprints and permission information is available at http://www.nature.com/reprints

Publisher's note Springer Nature remains neutral with regard to jurisdictional claims in published maps and institutional affiliations.

\begin{abstract}
cc) (i) Open Access This article is licensed under a Creative Commons Attribution 4.0 International License, which permits use, sharing, adaptation, distribution and reproduction in any medium or format, as long as you give appropriate credit to the original author(s) and the source, provide a link to the Creative Commons license, and indicate if changes were made. The images or other third party material in this article are included in the article's Creative Commons license, unless indicated otherwise in a credit line to the material. If material is not included in the article's Creative Commons license and your intended use is not permitted by statutory regulation or exceeds the permitted use, you will need to obtain permission directly from the copyright holder. To view a copy of this license, visit http://creativecommons.org/ licenses/by/4.0/.
\end{abstract}

(C) The Author(s) 2021 